\title{
Cytocompatibility and Antibacterial Properties of Capping Materials
}

\author{
Claudio Poggio, ${ }^{1}$ Carla Renata Arciola, ${ }^{2}$ Riccardo Beltrami, ${ }^{3}$ Annachiara Monaco, \\ Alberto Dagna, ${ }^{1}$ Marco Lombardini, ${ }^{1}$ and Livia Visai ${ }^{4,5}$ \\ ${ }^{1}$ Department of Clinical-Surgical, Diagnostic and Pediatric Sciences, University of Pavia, Policlinico "San Matteo", \\ Piazzale Golgi 3, 27100 Pavia, Italy \\ ${ }^{2}$ Research Unit on Implant Infections, Rizzoli Orthopedic Institute, DIMES of the University of Bologna Via di Barbiano 1/10, \\ 40136 Bologna, Italy \\ ${ }^{3}$ Department of Brain and Behavioral Sciences, University of Pavia, Via Bassi 21, 27100 Pavia, Italy \\ ${ }^{4}$ Department of Molecular Medicine, Center for Tissue Engineering (CIT), INSTM UdR of Pavia, University of Pavia, \\ Viale Taramelli 3/b, 27100 Pavia, Italy \\ ${ }^{5}$ Department of Occupational Medicine, Ergonomy and Disability, Laboratory of Nanotechnology, Salvatore Maugeri Foundation, \\ IRCCS, Via S. Boezio 28, 27100 Pavia, Italy
}

Correspondence should be addressed to Claudio Poggio; claudio.poggio@unipv.it

Received 24 February 2014; Accepted 24 April 2014; Published 18 May 2014

Academic Editor: Louis M. Lin

Copyright (C) 2014 Claudio Poggio et al. This is an open access article distributed under the Creative Commons Attribution License, which permits unrestricted use, distribution, and reproduction in any medium, provided the original work is properly cited.

The aim of this study was to evaluate and compare the antimicrobial activity and cytocompatibility of six different pulp-capping materials: Dycal (Dentsply), Calcicur (Voco), Calcimol LC (Voco), TheraCal LC (Bisco), MTA Angelus (Angelus), and Biodentine (Septodont). To evaluate antimicrobial activity, materials were challenged in vitro with Streptococcus mutans, Streptococcus salivarius, and Streptococcus sanguis in the agar disc diffusion test. Cytocompatibility of the assayed materials towards rat MDPC-23 cells was evaluated at different times by both MTT and apoptosis assays. Results significantly differed among the different materials tested. Both bacterial growth inhibition halos and cytocompatibility performances were significantly different among materials with different composition. MTA-based products showed lower cytotoxicity and valuable antibacterial activity, different from calcium hydroxide-based materials, which exhibited not only higher antibacterial activity but also higher cytotoxicity.

\section{Introduction}

Direct pulp-capping is a procedure for covering the exposed surface of the pulp to maintain its vitality and preserve its functional and biologic activities. The ultimate goal of capping material has been widely recognized as inducing pulp cells to form hard tissue [1].

Several materials such as calcium hydroxide-based materials and, more recently, mineral trioxide aggregate (MTA) are commonly recommended to seal communications between the exposed pulp and the oral cavity [2-6].

Calcium hydroxide-based materials are the most popular agents for direct and indirect pulp-capping, given their ability to release hydroxyl $(\mathrm{OH})$ and calcium $(\mathrm{Ca})$ ions upon dissolution [7-9]. It is assumed that they lead to initial change that causes undifferentiated cells within the pulp to differentiate into odontoblasts, which then form a hard tissue barrier at the pulp exposure site $[10,11]$. The formation of reparative dentine in response to calcium hydroxide may not be due to the bioinductive capacity of the material, but it is due to the result of a defense mechanism by the pulp induced by the irritant nature of calcium hydroxide [12-15].

Dycal (Dentsply, Milford, DE, USA) is a self-setting (2.5-3.5 $\mathrm{min}$ ) radiopaque calcium hydroxide-based material employed in direct and indirect pulp-capping procedures. Its alkaline $\mathrm{pH}$ ( $\mathrm{pH}$ 9-11) stimulates the formation of secondary dentine when the material is in direct contact with the pulp. Its toxicity to pulp cells is well documented [16]. 
Mineral trioxide aggregate (MTA) cement has a composition similar to that of Portland cement (PC). Both are composed of calcium phosphate, calcium, and silicon oxide. MTA, in addition, contains bismuth oxide, which provides radiopacity. MTA is a powder that contains trioxides and hydrophilic particles, which set in the presence of moisture [17].

MTA was first introduced as a water-based grey-colored root-end filling and perforation repair material [18]. It has high $\mathrm{pH}$ but low compressive strength; depending on its powder/liquid ratio, MTA possesses some antibacterial properties [19]. MTA seals well and is a biocompatible cement; hydroxyapatite crystals form over MTA in contact with tissue fluid $[17,20]$. However, it has some known drawbacks, such as a long setting time, high price, and potential discoloration [21]. Moreover, MTA cements exhibit calcified tissue-conductive activity and facilitate the differentiation of human orofacial mesenchymal stem cells [22] and the mineralization process in human dental pulp cells. They also have the potential to be used as pulp-capping materials.

MTA-Angelus (Angelus, Londrina, PR, Brazil) is bioactive, biocompatible, and self-setting hydrophilic calcium silicate cement $[17,19,22]$ now successfully used for direct pulp-capping $[21,23]$. It contains type III Portland cement, bismuth oxide, tricalcium silicate, dicalcium silicate, and tricalcium aluminate tetracalcium aluminoferrite. MTA is more effective and better than calcium hydroxide materials, as it has an enhanced interaction with dental pulp tissue [24] with limited pulp tissue necrosis (less caustic effect) shortly after its application and less pulp inflammation [25]. MTA facilitated the proliferation/differentiation of human dental pulp cells [24] and exhibited calcified tissue-conductive activity with the ability to stimulate faster complete dentine bridge formation and new hard tissue formation, thus revealing a material endowed with the potential for integration into the peri-implant tissues and preventing infection [25-28].

TheraCal (Bisco Inc., Schaumburg, IL, USA) is a new light-cured, resin-modified, calcium silicate-filled base/liner material designed with direct and indirect pulp-capping. It contains polymerizable methacrylate monomers, Portland cement type III, polyethylene glycol dimethacrylate, and barium zirconate. TheraCal is well tolerated by immortalized odontoblast cells [29].

Calcicur (Voco GmbH, Cuxhaven, Germany) is a readyto-use radiopaque water-based calcium hydroxide paste. It contains $45 \%$ calcium hydroxide and exhibits a high alkalizing $\mathrm{pH}$ correlating positively with the $\mathrm{Ca}(\mathrm{OH})_{2}$ mass fraction contained in it.

Calcimol LC (Voco GmbH, Cuxhaven, Germany) is a light-cured, resin-modified calcium ion releasing base liner and pulp-capping material. It contains urethane dimethacrylate resin, calcium dihydroxide, dimethylaminoethyl-methacrylate, and triethyleneglycol dimethacrylate (TEGDMA).

Biodentine (Septodont, Saint-Maur-des-Fosses, France) is a bicomponent material. The powder contains tricalcium silicate, calcium carbonate, and zirconium oxide; the liquid contains water, calcium chloride (accelerator), and modified polycarboxylate. Biodentine is an interesting alternative to conventional calcium hydroxide-based materials. It offers advantages for direct pulp-capping and, in properly selected cases, may contribute to the long-term maintenance of tooth vitality [30].

Among the properties that an endodontic sealer or a material for pulp-capping should have, the antibacterial activity can influence the success of the treatment. Furthermore, the materials that possess both optimum flow ability and antibacterial properties might theoretically eliminate residual microorganisms located around exposed pulp without damaging pulp tissue.

One of the objectives of operative dentistry is at the same time to maintain the pulp health in compromised teeth, thus reducing the need for root canal treatment and the potential for unwanted sequelae such as tooth loss. As reported above, methods used for this purpose are direct pulp-capping and pulpotomy, which consist of placement of biocompatible materials and bioinductors on the exposed pulp tissue to preserve its health and stimulate repair by mineralized tissue formation [2, 31]. A fundamental feature for these materials is biocompatibility, which includes antibacterial and healing induction properties, cytocompatibility, and sealing capabilities. As these materials will be in direct contact with pulp tissue for long periods, the biocompatibility is of a particular importance. A biocompatible material should not only promote tissue repair, but should also aid or stimulate the reorganization of injured structures [32, 33]. For the determination of biocompatibility of dental materials, a large number of methods have been recommended, with the analysis of cellular reactions in vitro being generally considered the initial approach [34].

One of the aims of this study was to evaluate and compare, by the agar disc diffusion test, the antimicrobial activity of different pulp-capping materials: Dycal (Dentsply), Calcicur (Voco), Calcimol LC (Voco), TheraCal LC (Bisco), MTA-Angelus (Angelus), and Biodentine (Septodont). In addition, the cytotoxicity of these pulp-capping cements on rat odontoblast-like MDPC-23 cells was assessed by both MTT and apoptosis assays.

\section{Materials and Methods}

2.1. Materials. Six pulp-capping materials were selected for this study: Dycal (Dentsply), Calcicur (Voco), Calcimol LC (Voco), TheraCal LC (Bisco), MTA Angelus (Angelus), and Biodentine (Septodont). Hydrogen peroxide was employed as a control. Table 1 shows chemical composition of the materials tested: they were prepared in strict compliance with the manufacturers' instructions.

2.2. Bacterial Strains and Growth Conditions. The streptococcal strains used in this study were from the Culture Collection of University of Goteborg (CCUG): Streptococcus mutans (CCUG 35176), Streptococcus salivarius (CCUG 11878), and Streptococcus sanguis (CCUG 17826). The cultures were grown and maintained in a Brain Heart Infusion (BHI, Difco, Detroit, MI, USA). S. mutans culture medium was supplemented with $10 \%(\mathrm{v} / \mathrm{v})$ heat-inactivated horse blood 
TABLE 1: Characteristics of tested materials.

\begin{tabular}{|c|c|c|c|}
\hline Material & Components & $\mathrm{pH}$ & Manufacturer \\
\hline Dycal & $\begin{array}{l}\text { Two-paste system made of a base paste (1,3-butylene glycol } \\
\text { disalicylate, zinc oxide, calcium phosphate, calcium tungstate, and } \\
\text { iron oxide pigments) and a catalyst paste (calcium hydroxide, } \\
\text { N-ethyl-o/p-toluene sulphonamide, zinc oxide, titanium oxide, zinc } \\
\text { stearate, and iron oxide pigments) }\end{array}$ & $9-11$ & $\begin{array}{l}\text { Dentsply Tulsa } \\
\text { Dental, Johnson } \\
\text { City, TN, USA }\end{array}$ \\
\hline Calcicur & Water-based calcium dihydroxide paste & 12.5 & $\begin{array}{l}\text { Voco GmbH, } \\
\text { Cuxhaven, } \\
\text { Germany }\end{array}$ \\
\hline Calcimol LC & $\begin{array}{l}\text { Light-curing radiopaque one-component material containing } \\
\text { urethane dimethacrylate resin, calcium dihydroxide, } \\
\text { dimethylaminoethyl-methacrylate, and TEGDMA }\end{array}$ & $10-12$ & $\begin{array}{l}\text { Voco GmbH, } \\
\text { Cuxhaven, } \\
\text { Germany }\end{array}$ \\
\hline TheraCal LC & $\begin{array}{l}\text { Light-curing, resin-modified calcium silicate filled liner single paste } \\
\text { containing } \mathrm{CaO} \text {, calcium silicate particles (type III Portland cement), } \\
\text { Sr glass, fumed silica, barium sulphate, barium zirconate, and resin } \\
\text { containing Bis-GMA and PEGDMA }\end{array}$ & $10-11$ & $\begin{array}{l}\text { Bisco Inc., } \\
\text { Schamburg, IL, } \\
\text { USA }\end{array}$ \\
\hline MTA-Angelus & $\begin{array}{l}\text { Powder containing type III Portland cement, bismuth oxide, } \\
\text { tricalcium silicate, dicalcium silicate, and tricalcium aluminate } \\
\text { tetracalcium aluminoferrite }\end{array}$ & 12 & $\begin{array}{c}\text { Angelus, Londrina, } \\
\text { PR, Brazil }\end{array}$ \\
\hline Biodentine & $\begin{array}{l}\text { Powder containing tricalcium silicate, calcium carbonate, and } \\
\text { zirconium oxide. } \\
\text { Liquid containing water, calcium chloride (accelerator), and modified } \\
\text { polycarboxylate }\end{array}$ & 12 & $\begin{array}{l}\text { Septodont, Saint- } \\
\text { Maur-des-Fosses, } \\
\quad \text { France }\end{array}$ \\
\hline
\end{tabular}

serum (Oxoid, Rodano, Milan, Italy) to improve its growth. The culture of all bacterial strains was statically incubated for $16 \mathrm{~h}$ at $37^{\circ} \mathrm{C}$ under aerobic conditions. This overnight culture, used as source for the experiments, was reduced at a final density of $1 \times 10^{10}$ cells $/ \mathrm{mL}$ as determined by comparing the $\mathrm{OD}_{600}$ of the sample with a standard curve relating $\mathrm{OD}_{600}$ to cell number.

\subsection{Antibacterial Test}

2.3.1. Agar Disc Diffusion Test. Sterile paper discs (diameter: $6 \mathrm{~mm}$, thickness: $1 \mathrm{~mm}$ ) from Whatman international, Maidstone, UK, were impregnated with $10 \mu \mathrm{L}$ of each pulpcapping material. All materials were prepared according to the manufacturers' recommendations. Hydrogen peroxide solution $\left(30 \% \mathrm{H}_{2} \mathrm{O}_{2}\right)$ was used as a positive control, whereas the paper disks not impregnated with any material (PD) were considered the negative control. Then, BHI-agar plates were incubated with $1 \times 10^{7}$ cells $/ \mathrm{mL}$ of an overnight culture of each streptococcal strain at $37^{\circ} \mathrm{C}$ for 20 minutes. Excess bacterial suspension was removed from the plates and incubated with the paper disks impregnated with the pulp-capping materials at $37^{\circ} \mathrm{C}$ for $24 \mathrm{~h}$. The diameter of the halo formed around the paper disc (inhibition zone) was measured by the same operator in two perpendicular locations with a millimeter ruler (sliding calliper) with an accuracy of $0.5 \mathrm{~mm}$, after $24 \mathrm{~h}$ and $48 \mathrm{~h}$. The size of the inhibition zone was calculated as follows:

size of inhibition zone

$$
=(\text { diameter of halo }- \text { diameter of specimen }) \times \frac{1}{2} \text {. }
$$

All the assays were conducted in triplicate and the results were recorded in terms of the average diameter of the inhibition zone.

\subsection{Cytotoxicity Assay}

2.4.1. Odontoblast Cell Line Culture Condition. The rat odontoblast-like cell line (MDPC-23) was kindly provided by Dr. Jacques Eduardo Nör (Dept. Cariology, Restorative Sciences, Endodontics; University of Michigan School of Dentistry). MDPC-23 cells were cultured in DMEM medium (Biowhittaker, Rome, Italy) supplemented with $10 \%$ fetal bovine serum (FBS), $2 \%$ glutamine, $2 \%$ sodium pyruvate, $1 \%$ amphotericin, and $1 \%(\mathrm{w} / \mathrm{v})$ streptomycin/penicillin at $37^{\circ} \mathrm{C}$ in $5 \% \mathrm{CO}_{2}$ atmosphere [35]. The cells were routinely detached using a trypsin-EDTA solution for $2 \mathrm{~min}$ at $37^{\circ} \mathrm{C}$ and resuspended in DMEM medium.

For the cytotoxicity tests, MDPC-23 cells were deposited in the lower chamber of the 24-well culture plate and left for $4 \mathrm{~h}$ at $37^{\circ} \mathrm{C}$ before any experiment.

2.5. Cytotoxicity Tests. We performed the cytotoxicity tests with the Transwell insert (Sigma-Aldrich, St. Louis, MO, USA) methodology and the immortalized rat odontoblast cell line MDPC-23. Cytotoxicity of the six pulp-capping materials was assessed with MDPC-23 cells grown in the lower chamber of a $24 \mathrm{~mm}$ diameter Transwell plate with a $0.3 \mathrm{~mm}$ pore size polycarbonate membrane (Sigma-Aldrich) [36].

Each pulp-capping material was mixed (Dycal (Dentsply), Calcicur (Voco), MTA Angelus (Angelus), and Biodentine (Septodont)) following the manufacturer's 
instructions onto paper disks or cured (Calcimol LC (Voco), TheraCal LC (Bisco)) by a halogen lamp (Elipar Trilight, $3 \mathrm{M}$-ESPE) for $20 \mathrm{~s}$ at $800 \mathrm{~mW} / \mathrm{cm}^{2}$, and all were placed in the Transwell membrane of the inner chamber.

The Transwell membrane of the inner chamber containing the pulp-capping materials was then placed into the lower chamber of the 24-well culture plate containing at the bottom $5 \times 10^{4}$ cells/well and incubated at $37^{\circ} \mathrm{C}$ in $5 \% \mathrm{CO}_{2}$ atmosphere for $24 \mathrm{~h}, 48 \mathrm{~h}$, and $72 \mathrm{~h}$, respectively. Some wells were incubated with only tissue culture media (negative control), whereas others were incubated with a $10 \%$ dilution of $30 \% \mathrm{H}_{2} \mathrm{O}_{2}$ (positive control). At the end of each incubation time the cell viability was performed with MTT test. The results were presented as percentage of cell viability with respect to cells incubated in absence of pulp-capping materials set at $100 \%$. The MDPC-23 treated with $\mathrm{H}_{2} \mathrm{O}_{2}$ did not show cell viability (data not shown). Five replicates for each pulp-capping material were used for each experiment performed in duplicate.

\subsection{3-(4,5-Dimethylthiazole-2-yl)-2,5-diphenyl Tetrazolium} Bromide (MTT) Test. To evaluate the mitochondrial activity of MDPC-23 cells, a test with 3-(4,5-dimethylthiazole-2-yl)2,5-diphenyl tetrazolium bromide (MTT; Sigma-Aldrich, St. Louis, MO, USA) was performed after $24 \mathrm{~h}, 48 \mathrm{~h}$, and $72 \mathrm{~h}$ as previously reported [37]. Aliquots of $200 \mu \mathrm{L}$ were sampled, and the related absorbance values were measured at $570 \mathrm{~nm}$ by a microplate reader (BioRad Laboratories, Hercules, CA, USA). A standard cell viability curve was used and the results were expressed as a percentage in relation to the untreated cells, respectively.

2.7. Apoptosis. An early event in apoptosis is the exposure of phosphatidylserine (PS) residues at the outer plasma membrane leaflet [38]. To determine the exposure of PS, cells were stained with an analog of Annexin V, PSVue480, according to the manufacturer's instructions (Molecular Targeting Technologies, West Chester, PA, USA). PSVue reagents are a family of fluorescent probes containing a bis(zinc ${ }^{2+}$ dipicolylamine) group (Zn-DPA), a motif that has been found to bind with high affinity to surfaces enriched with anionic phospholipids, especially phosphatidylserine (PS) exposed on cell membranes. Briefly, MDPC-23 cells were seeded on glass coverslips at a density of $5 \times 10^{4}$ cells per well and incubated with $\mathrm{H}_{2} \mathrm{O}_{2}$ (positive control; $100 \mathrm{mM}$ for $18 \mathrm{~h}$ ), without pulp-capping materials (negative control), and with each one of the six pulp-capping materials for $48 \mathrm{~h}$, respectively. At the end of each culture condition, cells were stained with PSVue480 solution prepared as follows: a $2 \mathrm{mM}$ solution of preweighed apo-PSS480 was prepared in DMSO until the solid apo-PSS480 was fully dissolved; an equal volume of $4.2 \mathrm{mM}$ zinc nitrate solution was then added; the resulting solution was placed in a water bath at $40^{\circ} \mathrm{C}$ and shaken frequently for $30 \mathrm{~min}$ to ensure complete complexation. A clear orange or red solution of $1 \mathrm{mM}$ stock in 1:1 DMSO/water resulted. The samples were stained with $10 \mu \mathrm{M}$ PSVue 480 by gently shaking for $2 \mathrm{~h}$ at room temperature and finally washed with $N$-tris(hydroxymethyl) methyl-2-aminoethane sulphonic acid buffer (TES). Then samples were counterstained with a propidium iodide solution $(2 \mu \mathrm{g} / \mathrm{mL})$ to target the cellular nuclei and then observed with a fluorescent microscope at 20x and 40x magnification.

2.8. Statistical Analysis. The diameter of the growth inhibition zones was analyzed by analysis of variance (ANOVA). Firstly, data were assessed to be normal by means of Kolmogorov and Smirnov test. The ANOVA and post hoc Tukey test were carried out. Significance was predetermined for $P<$ 0.001. Descriptive statistics, including mean, standard deviation, minimum, median, and maximum, were calculated for each group tested.

The distribution of the numbers of vital cells for every pulp-capping material was assessed to be normal with the Kolmogorov and Smirnov test. The data were then analyzed by ANOVA. Post hoc Bonferroni test was applied to investigate the differences among the number of vital cells of the materials. Significance was predetermined for $P<0.001$.

The analyses were conducted with Stata/SE 12.0 software (College Station, TX, USA).

\section{Results}

3.1. Antimicrobial Activity. The antimicrobial activity of the tested pulp-capping materials was evaluated with the agar disk diffusion test (Figure 1). As shown in Figure 1, the results were quite different among the three streptococcal strains and the pulp-capping materials. MTA-Angelus (Angelus), TheraCal LC (Bisco), Dycal (Dentsply), and Calcicur (Voco) showed a decreasing antibacterial effect on S. mutans; only Dycal (Dentsply) was effective against $S$. salivarius; Calcicur (Voco), Dycal (Dentsply), and Calcimol LC (Voco), followed by Biodentine (Septodont), were effective on S. sanguis. Dycal (Dentsply) was the only pulp-capping material showing a discreet antibacterial effect against all the three streptococcal strains.

For the investigation of the antibacterial properties, the ANOVA showed the presence of significant differences among the various groups. Tukey test showed that when testing antibacterial activity with Streptococcus salivarius, the highest growth inhibition values $(P<0.001)$ were reported with Dycal (Dentsply). MTA-Angelus (Angelus) showed significantly lower values than Dycal (Dentsply) and significantly higher values than all other pulp-capping materials $(P<0.001)$. Calcimol LC (Voco) and TheraCal LC (Bisco) showed no significant differences between them $(P>0.05)$ and all showed significantly lower values than Biodentine (Septodont) and Calcicur (Voco).

When testing antibacterial activity with Streptococcus sanguis, the highest growth inhibition values $(P<0.001)$ were reported with Dycal (Dentsply) and Calcicur (Voco). The lowest growth inhibition values $(P<0.001)$ were reported with MTA-Angelus (Angelus) and TheraCal LC (Bisco). Biodentine (Septodont) and Calcimol LC (Voco) showed significantly lower values than Dycal (Dentsply) and Calcicur (Voco) and significantly higher values than all other adhesives tested $(P<0.05)$. 


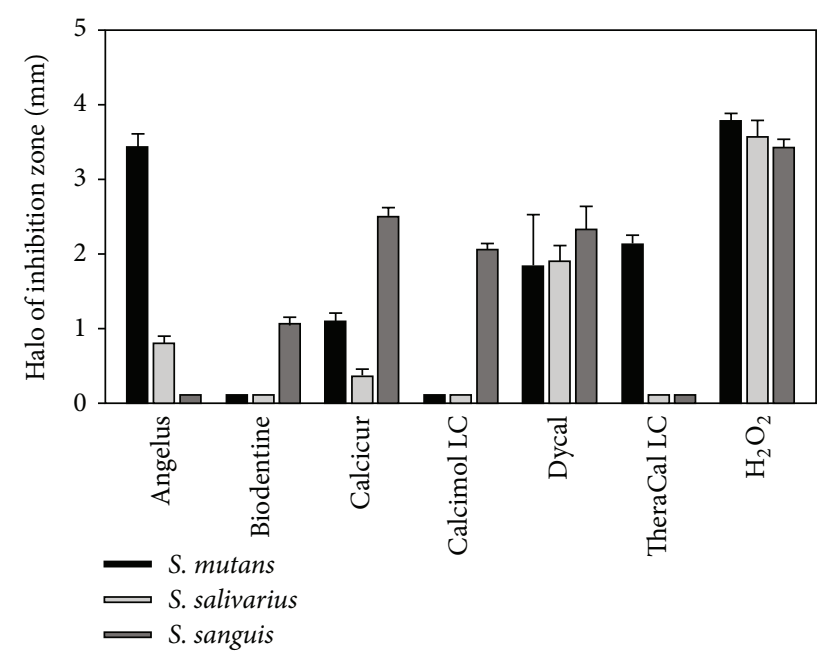

FIGURE 1: Antibacterial activity of the different pulp-capping materials evaluated by agar diffusion test. Each paper disk impregnated with the different pulp-capping materials was placed on agar plates previously incubated with the indicated streptococcal strains and incubated at $37^{\circ} \mathrm{C}$ for $24 \mathrm{~h}$. The positive control was represented by a $10 \%$ dilution of $30 \% \mathrm{H}_{2} \mathrm{O}_{2}$. All the assays were conducted in triplicate and the results were recorded in terms of the average diameter of inhibition zone $(\mathrm{mm})$. Error bars indicate standard errors of the means.

When testing antibacterial activity with Streptococcus mutans, the highest growth inhibition values were reported with MTA-Angelus (Angelus) $(P<0.001)$. Significantly lower values were reported with TheraCal LC (Bisco) and Dycal (Dentsply) that showed significantly higher values than Biodentine (Septodont), Calcimol LC (Voco), and Calcicur (Voco) $(P<0.05)$.

3.2. Cytocompatibility Study with MDPC-23 Cells. The cytocompatibility investigations were performed with all materials at different times of culture with MDPC-23 cells (Figure 2). As shown in Figure 2, Biodentine (Septodont) and MTA-Angelus (Angelus) showed the highest percent of cell cytocompatibility if compared to the other pulp-capping materials. Biodentine (Septodont) did not show any difference in cell viability at the three incubation times, whereas MTA-Angelus (Angelus) shows a lower cytocompatibility at $72 \mathrm{~h}$ (70\%); Calcicur (Voco) showed a discrete cell cytocompatibility (50-60\%) whereas Calcimol LC (Voco) and TheraCal LC (Bisco) show a very low cytocompatibility but only at longer incubation time $(72 \mathrm{~h})$. Dycal (Dentsply) showed the lowest cytocompatibility ( $10 \%$ cell viability) among the pulpcapping materials independently of the culture times.

At $24 \mathrm{~h}$, the highest numbers of viable cells were obtained with Biodentine (Septodont) and MTA-Angelus (Angelus) $(P<0.001)$. Calcimol LC (Voco), TheraCal LC (Bisco), and Calcicur (Voco) presented cytocompatibility values significantly lower than Biodentine (Septodont) and MTA-Angelus (Angelus). Dycal (Dentsply) showed the lowest cytocompatibility $(P<0.001)$.

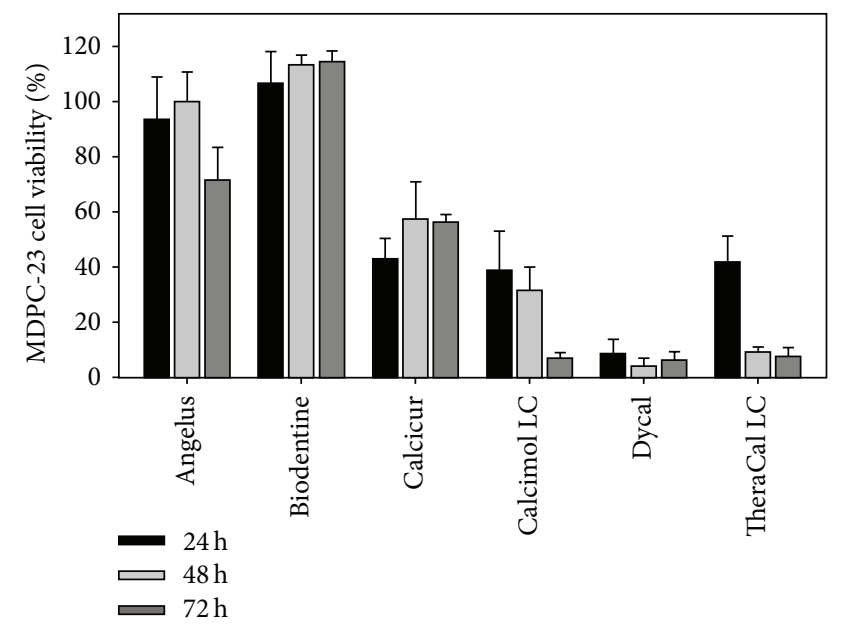

FIGURE 2: MDPC-23 cells cytocompatibility of the different pulpcapping materials using the Transwell method. MDPC-23 cells were incubated with the different pulp-capping materials at $37^{\circ} \mathrm{C}$ for $24 \mathrm{~h}$, $48 \mathrm{~h}$, and $72 \mathrm{~h}$ in a Transwell culture plate as reported in Materials and Methods Section. The cell viability was assessed with MMT test. The data are presented as percent of the control incubated in absence of any materials and set as $100 \%$. Five replicates for each pulp-capping material were used for each experiment performed in duplicate. Error bars indicate standard errors of the means.

After $48 \mathrm{~h}$, MTA-Angelus (Angelus) and Biodentine (Septodont) showed no significant differences in cytocompatibility $(P<0.001)$. The lowest cytocompatibility was shown by Dycal (Dentsply) and TheraCal LC (Bisco) $(P<$ 0.001), while Calcicur (Voco) and Calcimol LC (Voco) presented a cytocompatibility better than Dycal (Dentsply) and TheraCal LC (Bisco) $(P<0.001)$.

After $72 \mathrm{~h}$, the highest cytocompatibility was obtained with Biodentine (Septodont) $(P<0.001)$. MTA-Angelus (Angelus) showed a little lower cytocompatibility $(P<$ 0.001). The lowest cytocompatibility was obtained with Dycal (Dentsply), Calcimol LC (Voco), and TheraCal LC (Bisco) $(P<0.001)$ while Calcicur (Voco) showed an intermediate cytocompatibility $(P<0.001)$.

In Figure 3, the CLSM images representative of MDPC23 cells indirectly cultivated with the different pulp-capping materials for $24 \mathrm{~h}$ and stained with PSVue480 reagent to evaluate cell apoptosis are reported. In absence of any type of materials, the cells were not green fluorescent (Figure 3(a)) and the nuclei turned stained with Hoechst, which usually stains live cells. $\mathrm{H}_{2} \mathrm{O}_{2}$ is very cytotoxic and the cells stained with PSVue480 reagent are completely fluorescent in green (Figure 3(b)). The CLSM images obtained after incubation with different pulp-capping materials confirmed the MTT assay results: MTA-Angelus (Angelus) (Figure 3(c)) and Biodentine (Septodont) (Figure 3(d)) were not cytotoxic whereas Calcicur (Voco) (Figure 3(e)) showed some cells fluorescent in green; Calcimol LC (Voco) (Figure 3(f)) and TheraCal (Bisco) (Figure 3(h)) were very slightly cytotoxic if compared to the negative control (Figure 3(a)); a few cells were observed 


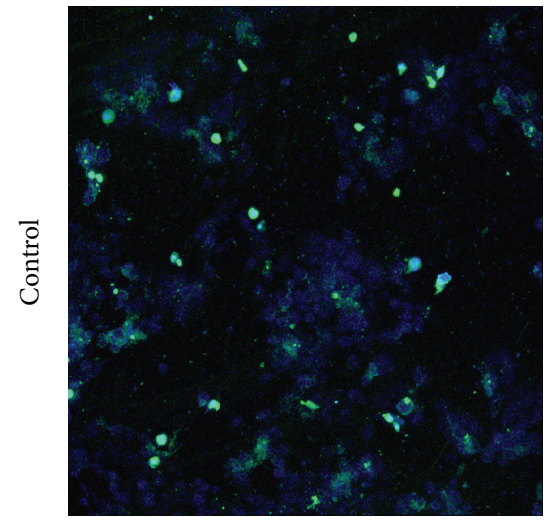

(a)

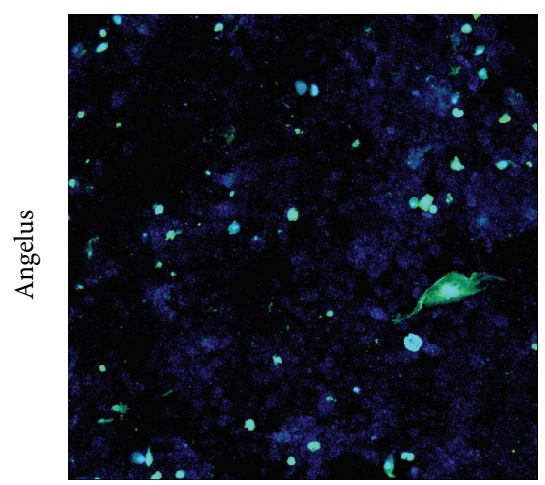

(c)

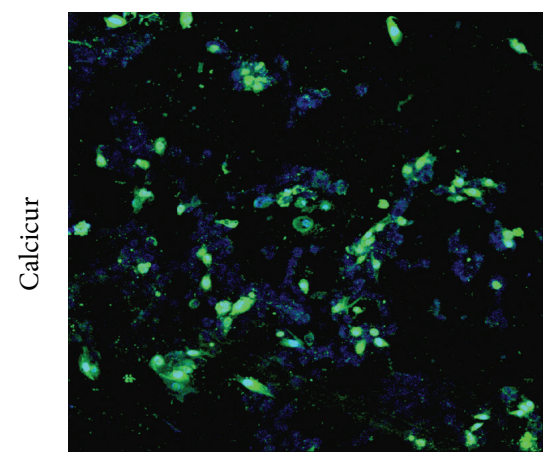

(e)

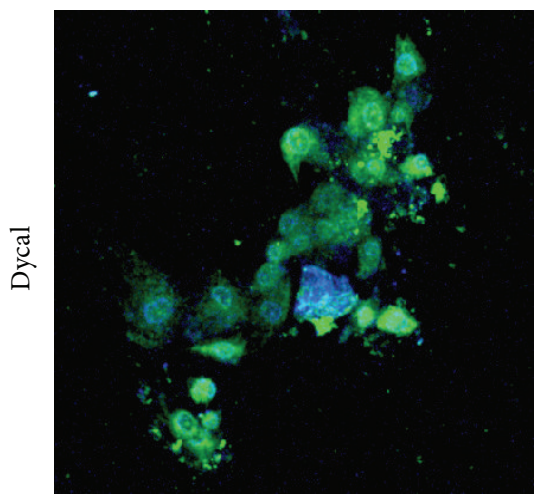

(g)

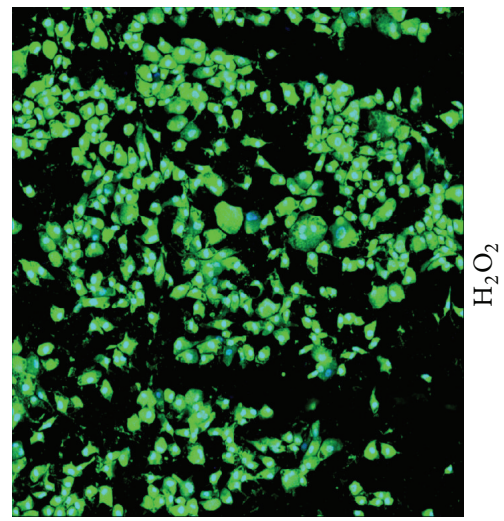

(b)

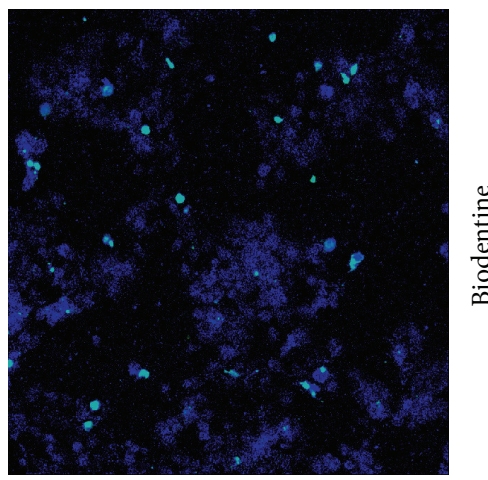

(d)

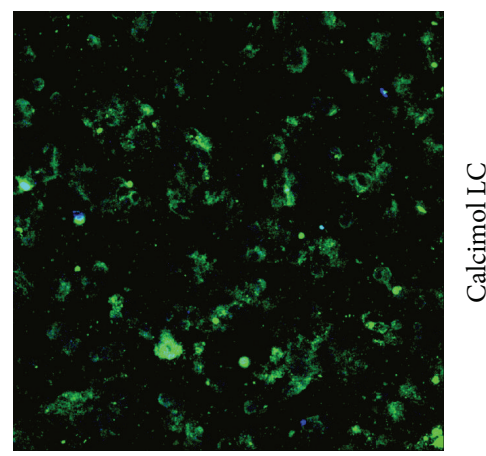

(f)

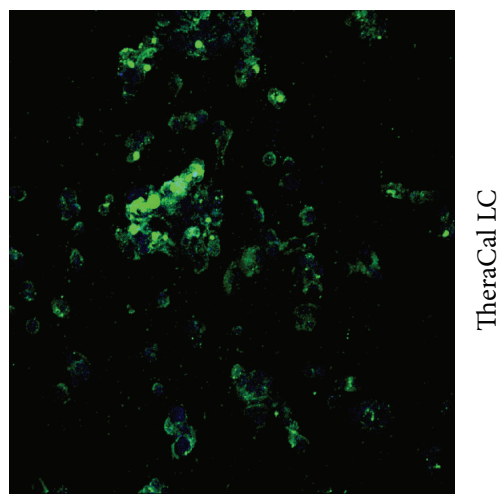

(h)

FIGURE 3: CLSM images of apoptosis assay. MDPC-23 cells were cultured in a 24-Transwell-tissue culture plate for $24 \mathrm{~h}$ at $37^{\circ} \mathrm{C}$ in the absence of any material (a) or in the presence of $\mathrm{H}_{2} \mathrm{O}_{2}$ (b), MTA Angelus (c), Biodentine (d), Calcicur (e), Calcimol LC (f), Dycal (g), and TheraCal LC (h). PSVue480 reagent was used for staining apoptotic cells. CLSM images were taken at 20x magnification. 
in the presence of Dycal (Dentsply), indicating a high level of cell cytotoxicity (Figure 3(g)).

At $24 \mathrm{~h}$, the highest numbers of viable cells were obtained with Biodentine (Septodont) and MTA-Angelus (Angelus) $(P<0.001)$. Calcimol LC (Voco), TheraCal LC (Bisco), and Calcicur (Voco) presented cytocompatibility values significantly lower than Biodentine (Septodont) and MTA-Angelus (Angelus). Dycal (Dentsply) showed the lowest cytocompatibility $(P<0.001)$.

After $48 \mathrm{~h}$, MTA-Angelus (Angelus) and Biodentine (Septodont) showed no significant differences in cytocompatibility $(P<0.001)$. The lowest cytocompatibility was shown by Dycal (Dentsply) and TheraCal LC (Bisco) $(P<$ 0.001), while Calcicur (Voco) and Calcimol LC (Voco) presented a cytocompatibility better than Dycal (Dentsply) and TheraCal LC (Bisco) $(P<0.001)$.

After $72 \mathrm{~h}$, the highest cytocompatibility was obtained with Biodentine (Septodont) $(P<0.001)$. MTA-Angelus (Angelus) showed lower cytocompatibility $(P<0.001)$. The lowest cytocompatibility was obtained with Dycal (Dentsply), Calcimol LC (Voco), and TheraCal LC (Bisco) $(P<0.001)$, while Calcicur (Voco) showed an intermediate cytocompatibility $(P<0.001)$.

\section{Discussion}

Microorganisms are considered the primary etiological agents in endodontic disease [39]. The agar diffusion test has been widely used to evaluate the antibacterial activity of dental materials [39-41]. The advantage of the agar diffusion test is that it allows direct comparisons of materials against the tested microorganisms, while a great disadvantage of this method is that it does not distinguish between microbiostatic and microbicidal properties of the materials [42]. Several factors that are relevant to the diffusion capacity of materials in agar must be considered, such as the contact between the experimental material and agar, molecular weight, size and shape of the antimicrobial agent, load and concentration of the test material, agar gel viscosity, and ionic concentration in relation to the medium. Furthermore, the control and standardization of the inoculation density, evaluation of results, selection of agar medium, incubation temperature of plates, and reading point of inhibition haloes are restricting factors affecting the dynamics and variability of diffusion tests in an agar medium [43].

Nevertheless, if most of these variables are carefully controlled, consistent and reproducible results may be obtained. Because of the obvious limitations of in vitro studies, clinical inferences should be drawn with strict caution. In our study, we obtained in vitro results that underline the antibacterial effects mainly for calcium hydroxide-based materials, as Dycal (Dentsply) and Calcicur (Voco). Similar results were obtained for Calcimol LC (Voco) and TheraCal LC (Bisco), both of which are light-curing materials, although the sensibility of the halo of inhibition zone was different among the species of microorganisms. MTA-based materials such as MTA-Angelus (Angelus) and Biodentine (Septodont) showed a variable effect against the different streptococci.
In the first step of our study on the antimicrobial effects of different pulp-capping materials, we confirmed that calcium hydroxide has an antibacterial activity, as reported in previous studies [44]. The antibacterial activity of $\mathrm{Ca}(\mathrm{OH})_{2}$ is related to the release of hydroxyl ions in an aqueous environment [45]. Hydroxyl ions are highly oxidant free radicals that show extreme reactivity with several biomolecules. This reactivity is high and indiscriminate, so this free radical rarely diffuses away from its site of generation [36]. However, this indiscriminate action also affects the mitochondrial activity of cultured cells when testing the cytocompatibility.

The antimicrobial effects of MTA-based materials are not yet well known. MTA consists of 50\% to 75\% (by weight) of calcium oxide and $15 \%$ to $25 \%$ of silicon dioxide. These two components together comprise $70 \%$ to $95 \%$ of the cement. When these raw materials are blended, they produce tricalcium silicate, dicalcium silicate, tricalcium aluminate, and tetracalcium aluminoferrite. When water is added, the cement hydrates to form silicate hydrate gel. It has been shown that, on hydration, MTA produces calcium hydroxide. Thus, it can be concluded that both MTA and calcium hydroxide may have a similar mechanism of action against bacteria [46].

Many studies have evaluated the effect of MTA on microorganisms, but with conflicting results [47-49]. Ribeiro et al. [50] suggested that these variations might be the results of the methodology used, such as aerobic and anaerobic incubations. It has been shown that in an aerobic atmosphere MTA can generate reactive oxygen species which, as reported above, have an antimicrobial activity similar to that obtained with calcium hydroxide. However, under anaerobic conditions, a decrease in the generation of radicals was observed [51]. Ribeiro et al. [50] reported that, in an anaerobic atmosphere, MTA was incapable of generating the radicals responsible for the antimicrobial effect on the different bacterial strains. In addition, Torabinejad et al. found that MTA had no antibacterial effect against any of the strict anaerobic bacteria [38]. However, as shown by our results, it is possible that MTA highly alkaline $\mathrm{pH}$ of 12.5 affords its antimicrobial activity [52] even when it acts in anaerobic conditions.

Exposing pulp tissue can cause inflammation and necrosis of the pulp itself, forcing the clinician to use an endodontic treatment. Pulp-capping materials should act as a barrier protecting the vitality of the entire pulp tissue by covering the minimal exposed tissue and by preventing the need for further endodontic treatments. Consequently, the material used should provide an appropriate host response. This means that tissues that are in contact with the materials must not present any toxic, irritating, inflammatory, allergic, genotoxic, or carcinogenic action [53]. In this study, we chose to use the Transwell insert methodology, which is a nondirect contact test [36] for the biocompatibility study. The advantage of using a nondirect contact test for the evaluation of the dental material cytotoxicity is related to the fact that cells and materials are usually separated. Furthermore, various different in vitro barrier tests have been already developed [54-57]. 
Our results indicate some considerable negative effects after the application of each of the materials tested on the culture plate, with the exception of Biodentine (Septodont). As shown in Figure 2, the decrease in the number of cells in the culture plate is sizeable for calcium hydroxide-based materials. Nonetheless, calcium hydroxide solutions have been largely used because of their property of stimulating dentin formation. In clinical practice, the presence of hard tissue barrier after capping can be considered an asset, since it provides natural protection against the infiltration of bacteria and chemical products [58]. However, the importance of calcified hard tissue barrier formation after capping has been challenged by other studies, which have shown multiple tunnel defects and cell inclusions in bridges following pulpcapping with calcium hydroxide $[59,60]$. This may lead to leakage and bacterial penetration into pulp tissue unlike the permanent seal produced by bonding agents. For both of these reasons, calcium hydroxide does not seem an eligible material to be used in the case of exposed pulp tissue [61]. In this study, the in vitro cytocompatibility analysis showed a better percentage of cell viability for MTA-based materials such as Biodentine (Septodont) and MTA-Angelus (Angelus), although an initial cytotoxic effect was recorded, which may be attributable to the high $\mathrm{pH}$ value of the composition of each material. During the $72 \mathrm{~h}$ of application of Biodentine (Septodont) on the culture plate, the modifications that occurred underlined the positive trend of the mitochondrial activity, although the differences between $24 \mathrm{~h}$ and $72 \mathrm{~h}$ were not statistically significant. The remnant calcium hydroxidebased materials showed a reduction in the percentage of cell viability after $72 \mathrm{~h}$, suggesting a different rate of cytocompatibility over the long-term period. In accordance with Hwang et al. [62], the present study emphasized the fact that MTAbased pulp-capping materials do not present cytotoxicity when tested by MTT assay. Other researchers using a shortterm in vivo assay [63], capable of generating well-founded clinical inferences, also confirm our in vitro results. In the last decade, many experimental and clinical studies have been carried out to develop and test new materials and new procedures endowed with safe biocompatibility and antiinfective properties [64-68].

\section{Conclusions}

MTA-based products show lower cytotoxicity and valuable antibacterial activity, unlike calcium hydroxide-based materials, which present not only higher antibacterial activity but also higher cytotoxicity. However, the conclusion that MTA-based pulp-capping material does not show cytotoxic effects in vitro should be taken with caution because the experimental design in vitro has some inevitable limitations with respect to the in vivo situation, where cellular responses and inflammatory and/or reparative reactions may differently influence the effects.

\section{Conflict of Interests}

The authors of this study have no conflict of interests to disclose.

\section{Acknowledgment}

The financial contribution by " 5 per mille" grants for Health Research to the Rizzoli Orthopedic Institute of Bologna is acknowledged.

\section{References}

[1] I. K. Bachoo, D. Seymour, and P. A. Brunton, "Biocompatible and bioactive replacement for dentine: is this a reality? The properties and uses of a novel calcium-based cement," British Dental Journal, vol. 214, no. 2, p. E5, 2013.

[2] M. S. Dominguez, D. E. Witherspoon, J. L. Gutmann, and L. A. Opperman, "Histological and scanning electron microscopy assessment of various vital pulp-therapy materials," Journal of Endodontics, vol. 29, no. 5, pp. 324-333, 2003.

[3] J. Camilleri and T. R. Pitt Ford, "Mineral trioxide aggregate: a review of the constituents and biological properties of the material," International Endodontic Journal, vol. 39, no. 10, pp. 747-754, 2006.

[4] C. Prati, F. Siboni, A. Polimeni, M. Bossu, and M. G. Gandolfi, "Use of calcium-containing endodontic sealers as apical barrier in fluid-contaminated wide-open apices.," Journal of Applied Biomaterials \& Functional Materials, 2014.

[5] S. M. Hasheminia, S. L. Nejad, O. Dianat, J. Modaresi, and F. Mahjour, "Comparing the sealing properties of mineral trioxide aggregate and an experimental ceramic based root end filling material in different environments," Indian Journal of Dental Research, vol. 24, no. 4, pp. 474-477, 2013.

[6] G. Malik, P. Bogra, S. Singh, and R. K. Samra, "Comparative evaluation of intracanal sealing ability of mineral trioxide aggregate and glass ionomer cement: an in vitro study," Journal of Conservative Dentistry, vol. 16, no. 6, pp. 540-545, 2013.

[7] S. Desai and N. Chandler, "The restoration of permanent immature anterior teeth, root filled using MTA: a review," Journal of Dentistry, vol. 37, no. 9, pp. 652-657, 2009.

[8] Z. Mohammadi and P. M. H. Dummer, "Properties and applications of calcium hydroxide in endodontics and dental traumatology," International Endodontic Journal, vol. 44, no. 8, pp. 697-730, 2011.

[9] P. Fulzele, S. Baliga, N. Thosar, and D. Pradhan, "Evaluation of calcium ion, hydroxyl ion release and $\mathrm{pH}$ levels in various calcium hydroxide based intracanal medicaments: an in vitro study," Contemporary Clinical Dentistry, vol. 2, no. 4, pp. 291295, 2011.

[10] P. Laurent, J. Camps, and I. About, "Biodentine induces TGF$\beta 1$ release from human pulp cells and early dental pulp mineralization," International Endodontic Journal, vol. 45, no. 5, pp. 439-448, 2012.

[11] O. Téclès, P. Laurent, V. Aubut, and I. About, "Human tooth culture: a study model for reparative dentinogenesis and direct pulp capping materials biocompatibility," Journal of Biomedical Materials Research B: Applied Biomaterials, vol. 85, no. 1, pp. 180-187, 2008.

[12] M. Goldberg, N. Six, F. Decup et al., "Bioactive molecules and the future of pulp therapy," The American Journal of Dentistry, vol. 16, no. 1, pp. 66-76, 2003.

[13] A. Almushayt, K. Narayanan, A. E. Zaki, and A. George, "Dentin matrix protein 1 induces cytodifferentiation of dental pulp stem cells into odontoblasts," Gene Therapy, vol. 13, no. 7, pp. 611-620, 2006. 
[14] H. H. Pinho Veloso, R. A. do Santos, T. P. de Araújo, D. P. Leonardi, and F. Baratto Filho, "Histological analysis of the biocompatibility of three different calcium hydroxide-based root canal sealers," Journal of Applied Oral Science, vol. 14, no. 5, pp. 376-381, 2006.

[15] A. Nosrat, A. Peimani, and S. Asgary, "A preliminary report on histological outcome of pulpotomy with endodontic biomaterials vs calcium hydroxide," Restorative Dentistry \& Endodontics, vol. 38, no. 4, pp. 227-233, 2013.

[16] A. Furey, J. Hjelmhaug, and D. Lobner, "Toxicity of flow line, durafill VS, and Dycal to dental pulp cells: effects of growth factors," Journal of Endodontics, vol. 36, no. 7, pp. 1149-1153, 2010.

[17] M. Torabinejad and M. Parirokh, "Mineral trioxide aggregate: a comprehensive literature review-part II: leakage and biocompatibility investigations," Journal of Endodontics, vol. 36, no. 2, pp. 190-202, 2010.

[18] M. Torabinejad, T. F. Watson, and T. R. Pitt Ford, "Sealing ability of a mineral trioxide aggregate when used as a root end filling material," Journal of Endodontics, vol. 19, no. 12, pp. 591-595, 1993.

[19] M. Parirokh and M. Torabinejad, "Mineral trioxide aggregate: a comprehensive literature review-part I: chemical, physical, and antibacterial properties," Journal of Endodontics, vol. 36, no. 1, pp. 16-27, 2010.

[20] S. Asgary, M. J. Eghbal, M. Parirokh, and J. Ghoddusi, "Effect of two storage solutions on surface topography of two root-end fillings," Australian Endodontic Journal, vol. 35, no. 3, pp. 147152, 2009.

[21] M. Parirokh and M. Torabinejad, "Mineral trioxide aggregate: a comprehensive literature review-part III: clinical applications, drawbacks, and mechanism of action," Journal of Endodontics, vol. 36, no. 3, pp. 400-413, 2010.

[22] M. G. Gandolfi, S. N. Shah, R. Feng, C. Prati, and S. O. Akintoye, "Biomimetic calcium-silicate cements support differentiation of human orofacial mesenchymal stem cells," Journal of Endodontics, vol. 37, no. 8, pp. 1102-1108, 2011.

[23] D. Tuna and A. Ölmez, "Clinical long-term evaluation of MTA as a direct pulp capping material in primary teeth," International Endodontic Journal, vol. 41, no. 4, pp. 273-278, 2008.

[24] T. Takita, M. Hayashi, O. Takeichi et al., "Effect of mineral trioxide aggregate on proliferation of cultured human dental pulp cells," International Endodontic Journal, vol. 39, no. 5, pp. 415-422, 2006.

[25] S. Moghaddame-Jafari, M. G. Mantellini, T. M. Botero, N. J. McDonald, and J. E. Nör, "Effect of ProRoot MTA on pulp cell apoptosis and proliferation in vitro," Journal of Endodontics, vol. 31, no. 5, pp. 387-391, 2005.

[26] T. Okiji and K. Yoshiba, "Reparative dentinogenesis induced by mineral trioxide aggregate: a review from the biological and physicochemical points of view," International Journal of Dentistry, vol. 2009, Article ID 464280, 12 pages, 2009.

[27] G. Bogen, J. S. Kim, and L. K. Bakland, "Direct pulp capping with mineral trioxide aggregate: an observational study," Journal of the American Dental Association, vol. 139, no. 3, pp. 305-315, 2008.

[28] C. R. Arciola, L. Montanaro, and J. W. Costerton, "New trends in diagnosis and control strategies for implant infections," International Journal of Artificial Organs, vol. 34, no. 9, pp. 727736, 2011.

[29] J. Hebling, F. C. R. Lessa, I. Nogueira, R. M. de Carvalho, and C. A. S. de Costa, "Cytotoxicity of resin-based light-cured liners,"
The American Journal of Dentistry, vol. 22, no. 3, pp. 137-142, 2009.

[30] M. Zanini, J. M. Sautier, A. Berdal, and S. Simon, "Biodentine induces immortalized murine pulp cell differentiation into odontoblast-like cells and stimulates biomineralization," Journal of Endodontics, vol. 38, no. 9, pp. 1220-1226, 2012.

[31] M. D. L. R. Accorinte, R. Holland, A. Reis et al., "Evaluation of mineral trioxide aggregate and calcium hydroxide cement as pulp-capping agents in human teeth," Journal of Endodontics, vol. 34, no. 1, pp. 1-6, 2008.

[32] T. L. Carvalho, J. M. Teófilo, C. A. Araújo, and L. G. Brentegani, "Chronology of alveolar healing following immediate implantation of Ricinus communis polyurethane resin: histometric analysis in rats," Journal of Biomedical Materials Research, vol. 37, no. 4, pp. 449-452, 1997.

[33] M. G. Mantellini, T. M. Botero, P. Yaman, J. B. Dennison, C. T. Hanks, and J. E. Nör, "Adhesive resin induces apoptosis and cellcycle arrest of pulp cells," Journal of Dental Research, vol. 82, no. 8, pp. 592-596, 2003.

[34] G. Schmalz and H. Schweikl, "Characterization of an in vitro dentin barrier test using a standard toxicant," Journal of Endodontics, vol. 20, no. 12, pp. 592-594, 1994.

[35] C. G. de Souza, N. S. Girardo, M. A. Costa, and R. M. Peralta, "Influence of growth conditions on the production of xylanolytic enzymes by Aspergillus flavus," Journal of Basic Microbiology, vol. 39, no. 3, pp. 155-160, 1999.

[36] H. Babich and M. C. Sinensky, "Indirect cytotoxicity of dental materials: a study with Transwell inserts and the neutral red uptake assay," Alternatives to Laboratory Animals, vol. 29, no. 1, pp. 9-13, 2001.

[37] E. Saino, S. Grandi, E. Quartarone et al., "In vitro calcified matrix deposition by human osteoblasts onto a zinc-containing bioactive glass," European Cells \& Materials, vol. 21, pp. 59-72, 2011.

[38] M. van Engeland, L. J. W. Nieland, F. C. S. Ramaekers, B, Schutte, and C. P. M. Reutelingsperger, "Annexin V-affinity assay: a review on an apoptosis detection system based on phosphatidylserine exposure," Cytometry, vol. 31, no. 1, pp. 1-9, 1998.

[39] Z. Z. Al-Khatib, R. H. Baum, D. R. Morse, C. Yesilsoy, S. Bhambhani, and M. L. Furst, "The antimicrobial effects of various endodontic sealers," Oral Surgery Oral Medicine and Oral Pathology, vol. 70, no. 6, pp. 784-790, 1990.

[40] S. Cohen and R. C. Burns, Pathways of the Pulp, Mosby, St. Louis, Mo, USA, 8th edition, 2002.

[41] F. K. Çobankara, H. C. Altinöz, O. Erganiş, K. Kav, and S. Belli, "In vitro antibacterial activities of root-canal sealers by using two different methods," Journal of Endodontics, vol. 30, no. 1, pp. 57-60, 2004.

[42] R. S. Tobias, "Antibacterial properties of dental restorative materials: a review," International Endodontic Journal, vol. 21, no. 2, pp. 155-160, 1988.

[43] C. C. Lai, F. M. Huang, H. W. Yang et al., "Antimicrobial activity of four root canal sealers against endodontic pathogens," Clinical oral investigations, vol. 5, no. 4, pp. 236-239, 2001.

[44] Y. Lu, T. Liu, H. Li, and G. Pi, "Histological evaluation of direct pulp capping with a self-etching adhesive and calcium hydroxide on human pulp tissue," International Endodontic Journal, vol. 41, no. 8, pp. 643-650, 2008.

[45] J. F. Siqueira Jr., "Strategies to treat infected root canals," Journal of the California Dental Association, vol. 29, no. 12, pp. 825-837, 2001. 
[46] H. W. Roberts, J. M. Toth, D. W. Berzins, and D. G. Charlton, "Mineral trioxide aggregate material use in endodontic treatment: a review of the literature," Dental Materials, vol. 24, no. 2, pp. 149-164, 2008.

[47] M. Torabinejad, C. U. Hong, T. R. P. Ford, and J. D. Kettering, "Antibacterial effects of some root end filling materials," Journal of Endodontics, vol. 21, no. 8, pp. 403-406, 1995.

[48] A. U. Eldeniz, H. H. Hadimli, H. Ataoglu, and D. Ørstavik, "Antibacterial effect of selected root-end filling materials," Journal of Endodontics, vol. 32, no. 4, pp. 345-349, 2006.

[49] K. Al-Hezaimi, T. A. Al-Shalan, J. Naghshbandi, S. Oglesby, J. H. S. Simon, and I. Rotstein, "Antibacterial effect of two Mineral Trioxide Aggregate (MTA) preparations against Enterococcus faecalis and Streptococcus sanguis in vitro," Journal of Endodontics, vol. 32, no. 11, pp. 1053-1056, 2006.

[50] C. S. Ribeiro, M. F. Z. Scelza, R. Hirata Jünior, and L. M. B. de Oliveira, "The antimicrobial activity of gray-colored mineral trioxide aggregate (GMTA) and white-colored MTA (WMTA) under aerobic and anaerobic conditions," Oral Surgery, Oral Medicine, Oral Pathology, Oral Radiology and Endodontology, vol. 109, no. 6, pp. e109-e112, 2010.

[51] E. Cabiscol, J. Tamarit, and J. Ros, "Oxidative stress in bacteria and protein damage by reactive oxygen species," International Microbiology, vol. 3, no. 1, pp. 3-8, 2000.

[52] E. G. Reston and C. A. de Souza Costa, "Scanning electron microscopy evaluation of the hard tissue barrier after pulp capping with calcium hydroxide, mineral trioxide aggregate (MTA) or ProRoot MTA," Australian Endodontic Journal, vol. 35, no. 2, pp. 78-84, 2009.

[53] J. W. Vahey, P. T. Simonian, and E. U. Conrad III, "Carcinogenicity and metallic implants," The American Journal of Orthopedics, vol. 24, no. 4, pp. 319-324, 1995.

[54] G. Schmalz, "A cell culture method for screening the biocompatibility of dental materials," in Biomaterials, G. D. Winter, D. F. Gibbons, and H. Plenk Jr., Eds., pp. 321-326, John Wiley \& Sons, New York, NY, USA, 1982.

[55] G. Schmalz, P. Garhammer, and H. Schweiki, "A commercially available cell culture device modified for dentin barrier tests," Journal of Endodontics, vol. 22, no. 5, pp. 249-252, 1996.

[56] G. Schmalz, "Agar overlay method," International Endodontic Journal, vol. 21, no. 2, pp. 59-66, 1988.

[57] A. Wennberg, G. Hasselgren, and L. Tronstad, "A method for toxicity screening of biomaterials using cells cultured on millipore filters," Journal of Biomedical Materials Research, vol. 13, no. 1, pp. 109-120, 1979.

[58] R. Holland, V. de Souza, W. de Mello, M. J. Nery, P. F. Bernabé, and J. A. Otoboni Filho, "Permeability of the hard tissue bridge formed after pulpotomy with calcium hydroxide: a histologic study," The Journal of the American Dental Association, vol. 99, no. 3, pp. 472-475, 1979.

[59] F. Goldberg, E. J. Massone, and C. Spielberg, "Evaluation of the dentinal bridge after pulpotomy and calcium hydroxide dressing," Journal of Endodontics, vol. 10, no. 7, pp. 318-320, 1984.

[60] J. C. Pereira, A. D. Segala, and C. A. S. Costa, "Human pulpal response to direct pulp capping with an adhesive system," The American Journal of Dentistry, vol. 13, no. 3, pp. 139-147, 2000.

[61] H. R. Stanley and C. H. Pameijer, "Dentistry's friend: calcium hydroxide," Operative Dentistry, vol. 22, no. 1, pp. 1-3, 1997.

[62] Y.-C. Hwang, S.-H. Lee, I.-N. Hwang et al., "Chemical composition, radiopacity, and biocompatibility of Portland cement with bismuth oxide," Oral Surgery, Oral Medicine, Oral Pathology,
Oral Radiology and Endodontology, vol. 107, no. 3, pp. e96-e102, 2009.

[63] T. Coutinho-Filho, G. De-Deus, L. Klein, G. Manera, C. Peixoto, and E. D. Gurgel-Filho, "Radiopacity and histological assessment of Portland cement plus bismuth oxide," Oral Surgery, Oral Medicine, Oral Pathology, Oral Radiology and Endodontology, vol. 106, no. 6, pp. e69-e77, 2008.

[64] C. R. Arciola, L. Montanaro, A. Moroni, M. Giordano, A. Pizzoferrato, and M. E. Donati, "Hydroxyapatite-coated orthopaedic screws as infection resistant materials: in vitro study," Biomaterials, vol. 20, no. 4, pp. 323-327, 1999.

[65] C. R. Arciola, D. Campoccia, P. Speziale, L. Montanaro, and J. W. Costerton, "Biofilm formation in Staphylococcus implant infections. A review of molecular mechanisms and implications for biofilm-resistant materials," Biomaterials, vol. 33, no. 26, pp. 5967-5982, 2012.

[66] D. Campoccia, L. Montanaro, and C. R. Arciola, "A review of the clinical implications of anti-infective biomaterials and infection-resistant surfaces," Biomaterials, vol. 34, no. 33, pp. 8018-8029, 2013.

[67] A. D. Pye, D. E. A. Lockhart, M. P. Dawson, C. A. Murray, and A. J. Smith, "A review of dental implants and infection," Journal of Hospital Infection, vol. 72, no. 2, pp. 104-110, 2009.

[68] J. D. Bumgardner, P. Adatrow, W. O. Haggard, and P. A. Norowski, "Emerging antibacterial biomaterial strategies for the prevention of peri-implant inflammatory diseases," International Journal of Oral \& Maxillofacial Implants, vol. 26, no. 3, pp. 553-560, 2011. 


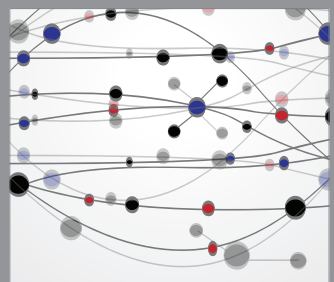

The Scientific World Journal
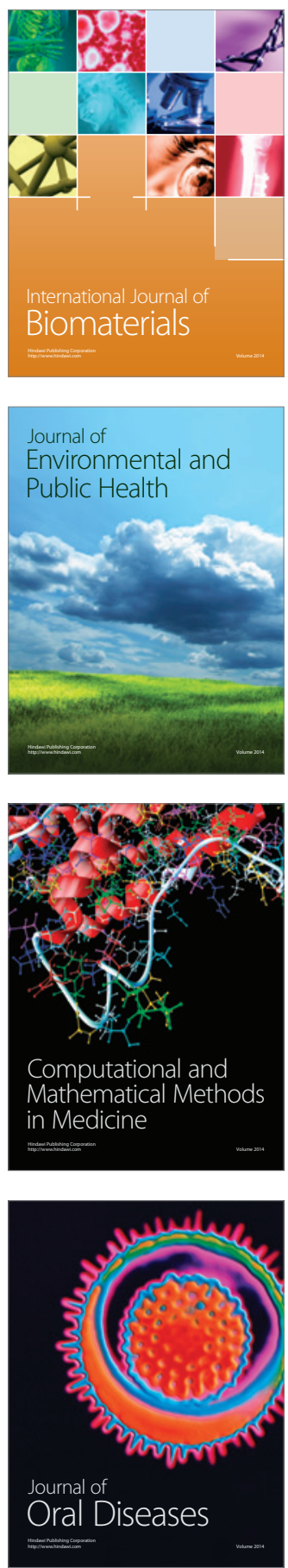
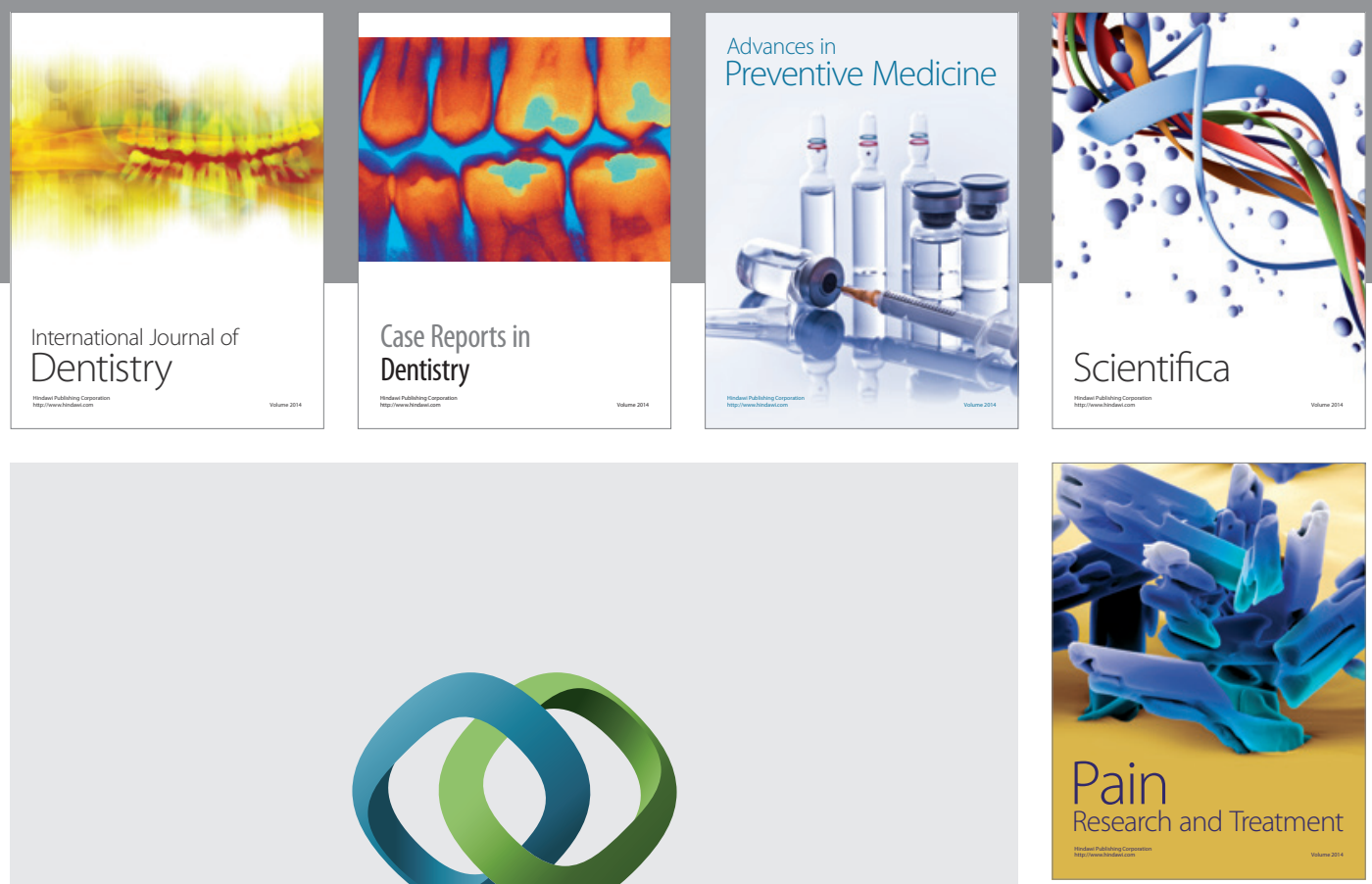

\section{Hindawi}

Submit your manuscripts at

http://www.hindawi.com
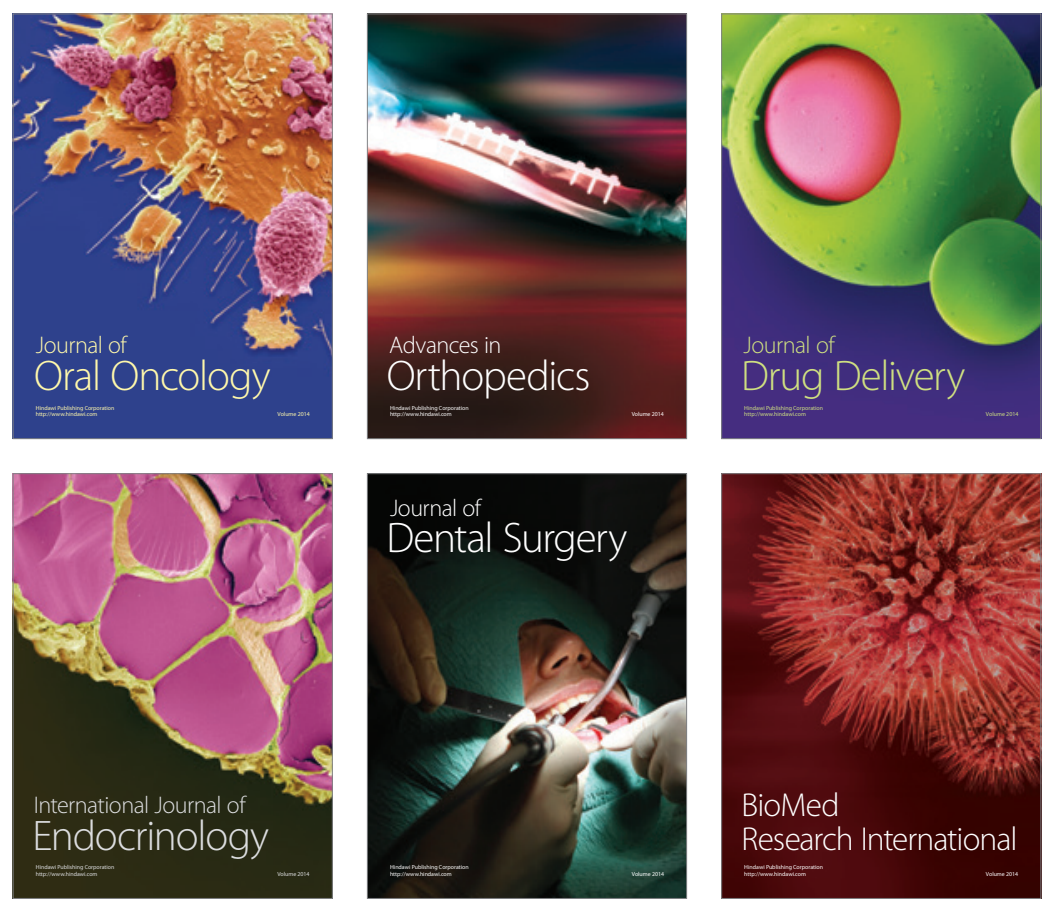

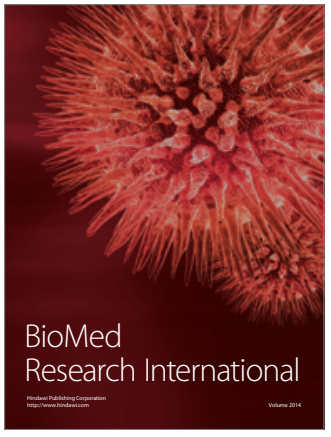

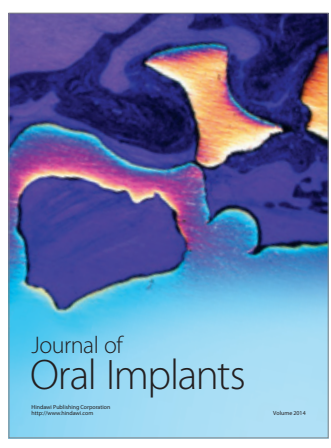
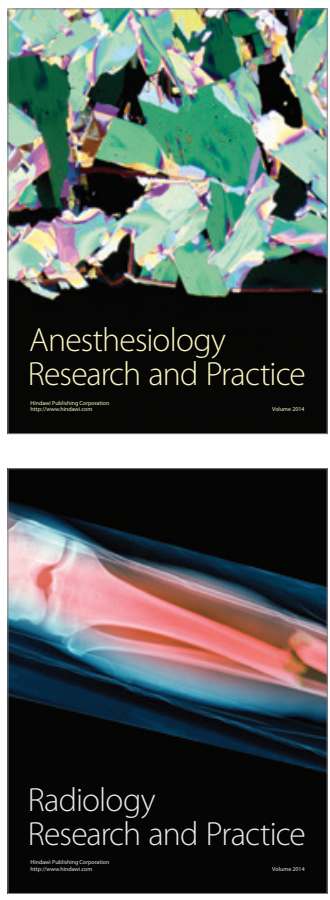